\title{
Regioselective oxidative fragmentation of drimanic terpene alcohols: a short, easy and efficient access to natural and synthetic 11-nordrimane terpene derivatives
}

\author{
Mauricio A. Cuellar, Luis E. Moreno, and Marcelo D. Preite* \\ Departamento de Química Orgánica, Facultad de Química, Pontificia Universidad Católica de \\ Chile, Vicuña Mackenna 4860, Casilla 306, Correo 22, Santiago, Chile \\ E-mail:mpreite@puc.cl \\ Dedicated to Professor Edmundo A. Rúveda on his $70^{\text {th }}$ birthday, \\ and to Professor Roberto A. Rossi on his $60^{\text {th }}$ birthday \\ (received 07 Jul 03; accepted 22 Aug 03; published on the web 26 Aug 03)
}

\begin{abstract}
Using selective Cr(VI) oxidations with Jones reagent and PCC, a regioselective and direct access to different oxygenated 11-nordrimane skeletons was achieved, in good overall yield, starting from naturally occurring drimane terpenes.
\end{abstract}

Keywords: 11-Nordrimanes, chromium oxidation, Jones reagent, PCC

\section{Introduction}

There is a large collection of natural drimanic sesquiterpenes, many of them showing a wide range of biological activities and potential industrial applications. ${ }^{1-6}$ For that reason, many efforts have been devoted to the syntheses of drimanes, usually starting from abundant, naturally occurring compounds. ${ }^{7}$

In marked contrast, there is hardly any natural product with an 11-nordrimane skeleton known to date, being most of them those shown in Figure 1: isonordrimenone (1), obtained in 1973 from several species of tobacco, as for instance, Greek tobacco, Nicotiana tabacum L., ${ }^{8}$ isopolygonal (2) and polygonone (3), obtained in 1985 from the leaves of the folk medicinal plant Polygonum hydropiper L., ${ }^{9}$ and polygonal (4), also isolated from Polygonum hydropiper L. in $1979 .{ }^{10}$ Very recently, in 2000, aldehyde 5 was isolated from the stem bark of the conifer tree Thuja standishii (Gord.)Carr. ${ }^{11}$

Interestingly, transposed 9-oxo compounds like $\mathbf{6}$ and $\mathbf{7}$ were never obtained from a natural source. Nevertheless, both carbonyl compounds found use as synthetic key intermediates in the construction of more complex substances: $\alpha, \beta$-unsaturated ketone $\mathbf{6}$ was recently used as a key 
intermediate in the synthesis of hispanone, ${ }^{12}$ and cross-conjugated keto-aldehyde 7 (together with its synthetic equivalents) was used in the synthesis of the feeding-deterrent natural compound warburganal. ${ }^{13,14}$

Here, we wish to report the regioselective preparation of $\mathbf{1}$ and $\mathbf{6}$, in one step from naturally occurring drimenol (8), and of $\mathbf{3}$ and $\mathbf{7}$, starting from diol 9, itself prepared by carbonyl reduction of naturally occurring, commercially available polygodial (10). ${ }^{16-20}$ Both 8 and $\mathbf{1 0}$ were easily obtained from the stem bark of South American Winter's bark tree. ${ }^{14,15}$

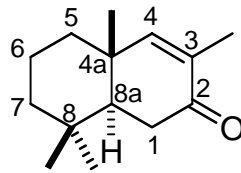

1<smiles>C[C@H]1CCCC2(C)C=C(C=O)[C@@H](O)C[C@]12C</smiles>

2<smiles>C[C@H]1CCCC2(C)C=C(C=O)C(=O)C[C@]12C</smiles>

3<smiles>CC1(C)CCCC2(C)C=C(C=O)[C@@H](O)C[C@@H]12</smiles>

4<smiles>CC12C=C(C=O)CC[C@@H]1[C@@](C)(C(=O)O)CCC2</smiles><smiles>CC1=CC[C@]2(C)[C@@H](C)CCC[C@]2(C)[C@@H]1CO</smiles><smiles>CC1(C)CCCC(C)(C)C12CC=C(CO)C(CO)C2</smiles>

9<smiles>[3H][C@H]1CCCC2(C)C(=O)C(C=O)=CC[C@H]12</smiles>

Figure 1. Natural and synthetic 11-nordrimanes and drimanes.

\section{Results and Discussion}

Compounds $\mathbf{8}$ and $\mathbf{1 0}$ were obtained by multigram preparative column chromatography (hexane: ethyl acetate gradient) from the crude hexane extract of the ground stem bark of Drimys winteri $F$., as previously described. ${ }^{14-20}$

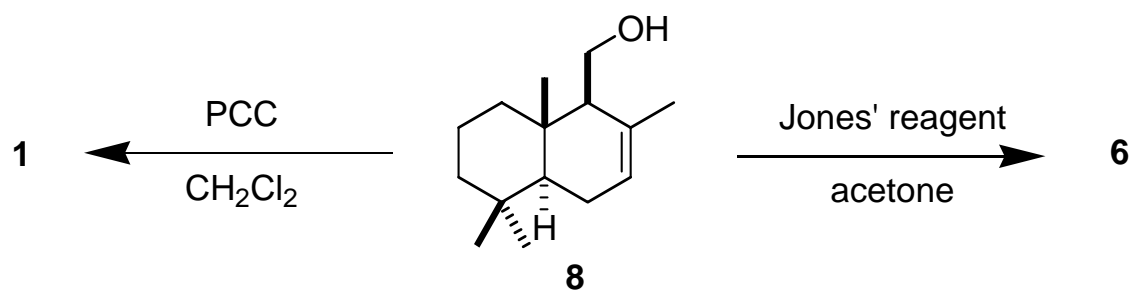


Scheme 1. Oxidation of drimenol (8).

We found that when an acetone solution of alcohol 8 was titrated with Jones reagent at $0^{\circ} \mathrm{C}$, unsaturated ketone 6 was obtained as a single compound (71\% yield, Scheme 1). On the other hand, when alcohol 8 was oxidized instead with pyridinium chlorochromate (PCC) suspended in dichloromethane at room temperature, unsaturated ketone 1 (65\% isolated yield) was obtained as a single compound (Scheme 1). The identities of enones $\mathbf{1}^{8,21,22}$ and $\mathbf{6}^{12,21,23}$ were established by comparison with the previously reported physical data for the same compounds.

In order to have access to compounds $\mathbf{3}$ and 7, it was possible to perform an allylic oxidation of 1 and 6, but we felt that a better and more convenient way would be to start with natural dialdehyde $\mathbf{1 0}$ (which is available to us from the natural source in better yield than alcohol $\mathbf{8}$ itself). Reduction of $\mathbf{1 0}$ with sodium borohydride in methanol at room temperature produced diol 9 in an excellent yield (Scheme 2). ${ }^{24,25}$ It was previously reported that treatment of diol 9 with one equivalent of tert-butyl dimethyl chlorosilane monoprotected specifically the C-12 hydroxy position. $^{24}$ In our case, protection of diol 9 with one equivalent of tert-butyl, diphenylsilyl chloride in DMF, in the presence of imidazole, produced silyl ether 11, with identical specificity towards the allylic alcohol, and excellent yield.

10
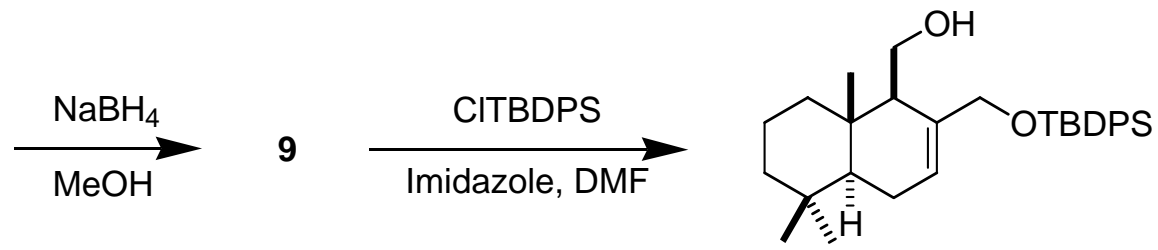

11

Scheme 2. Preparation of monoprotected alcohol 11.

Unsaturated alcohol 11 was submitted to oxidation with Jones reagent (Scheme 3), affording enone 12 with a moderate yield. Deprotection of silyl-ether 12 with tetrabutylammonium fluoride in anhydrous tetrahydrofuran furnished alcohol 13 quantitatively, and final mild oxidation of 13 with pyridinium chlorochromate gave ketoaldehyde 7 (27\% overall yield from 10).

11

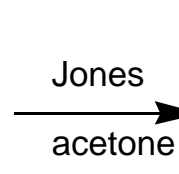

TBAF, THF<smiles>[R]CC1=CC[C@]2(C)[C@@H](C)CCC[C@]2(C)C1=O</smiles>

12: $R=$ TBDPS

13: $\mathrm{R}=\mathrm{H}$
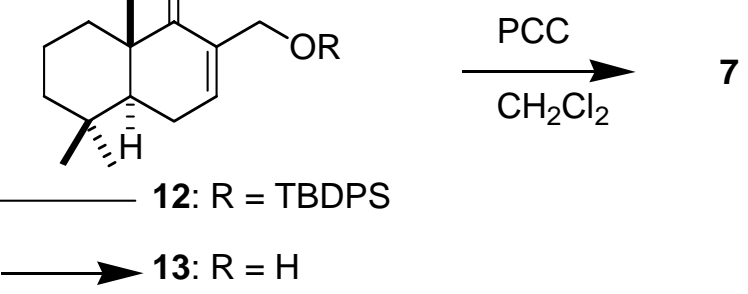
Scheme 3. Jones oxidation of alcohol 11, and conversion to enone 7.

When alcohol 11 was treated instead with pyridinium chlorochromate (Scheme 4), it produced instead enone 14. Deprotection of silylated ketone 14 with tetrabutylammonium fluoride in anhydrous tetrahydrofuran furnished alcohol 15 quantitatively, and finally, oxidation of $\mathbf{1 5}$ with pyridinium chlorochromate gave access ketoaldehyde 3 (37\% overall yield from 10).

11

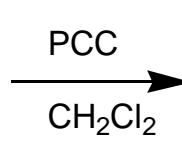

TBAF, THF

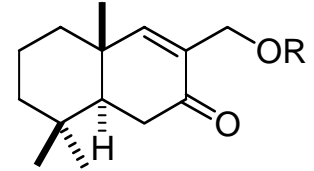

14: $R=$ TBDPS

15: $\mathrm{R}=\mathrm{H}$

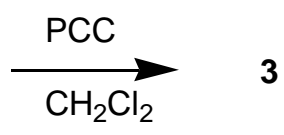

3

Scheme 4. PCC oxidation of alcohol 11, and conversion to enone 3.

\section{Conclusions}

In summary, we described here a simple methodology for the degradation of drimane alcohols to the corresponding 11-nordrimane enones or ketoaldehydes, with total control of the regiospecificity of the reaction. Specifically, we prepared natural isonordrimenone (1) with a $71 \%$ overall yield and polygonone (3), with a 37\% overall yield, and their synthetic regioisomers 6, obtained with a $65 \%$ overall yield, and 7 , prepared with a $27 \%$ overall yield (all yields calculated from the natural drimanic starting material). Compounds $\mathbf{1}$ and $\mathbf{6}$ were previously isolated from natural sources, and compounds $\mathbf{3}$ and $\mathbf{7}$ were previously used as key intermediates in the total synthesis of complex natural compounds. Due to the easy availability of our starting materials from the chiral pool, and the good overall yields obtained in these short preparations, we believe that these compounds could find in future a good use in the stereoselective synthesis of complex bioactive molecules, even at the industrial scale. A study of their synthetic applications, is currently under way in our labs.

\section{Experimental Section}

General Procedures. All reactions were routinely run under a dry nitrogen atmosphere, with flame-dried glassware, and with magnetic stirring. All chemicals were used as purchased.

Melting points were determined in a Stuart Scientific Apparatus SMP3, and are uncorrected. Optical rotations were measured in $\mathrm{CHCl}_{3}$ solutions, in a $0.1 \mathrm{dm}$ cell, in an Optical Activity, Ltd instrument. Infrared spectra were recorded in a Bruker Vector-22 FTIR spectrometer. NMR Spectra were obtained on a Bruker AC 200P (200.13 MHz for ${ }^{1} \mathrm{H}, 50.13 \mathrm{MHz}$ for ${ }^{13} \mathrm{C}$ ) or Avance 
400 (400.13 MHz for ${ }^{1} \mathrm{H}, 100.13 \mathrm{MHz}$ for ${ }^{13} \mathrm{C}$ ) spectrometer, in $\mathrm{CDCl}_{3}$ solutions with TMS as internal standard. For 2D, COSY, NOE, HSQC and HMBC experiments, Bruker standard software was used. Assignments were done by a combination of 1D and 2D NMR techniques, in each case, as needed. The symbol * was used to denote signal pairs with interchangeable assignments.

For skeleton carbon numberings, please refer to Figure 1. Elemental analyses were obtained in a Fisons Instruments EA 1108 microanalyzer. Column chromatography was performed on silica gel $60 \mathrm{H}$, slurry packed, run under low pressure of air, and employing increasing amounts of ethyl acetate in hexane as solvent. Analytical TLC was carried out using Kieselgel Merck $\mathrm{F}_{254}$ with thickness $0.20 \mathrm{~mm}$.

Preparation of (5S, 10S)-(9 $\alpha H)$-7-drimene-11,12-diol (9). To a magnetically well-stirred solution of polygodial (10, $3.4 \mathrm{~g}, 14.53 \mathrm{mmol})$ in methanol $(50 \mathrm{ml})$, sodium borohydride $(2.0 \mathrm{~g}$, $53 \mathrm{mmol}$ ) was added in small portions and carefully. The reaction mixture was stirred at room temperature for an additional $2 \mathrm{~h}$, water $(20 \mathrm{ml})$ was added cautiously, and most of the solvent was evaporated in vacuo. The resulting slurry was diluted with ethyl acetate $(100 \mathrm{ml})$, washed with water $(50 \mathrm{ml}), 1 \mathrm{~N}$ hydrochloric acid $(50 \mathrm{ml})$, and water $(50 \mathrm{ml})$ again, dried $\left(\mathrm{MgSO}_{4}\right)$, and evaporated. The oily yellowish residue was purified by column chromatography, to give diol $\mathbf{9}$ (3.1g, 90\%), as a white solid: m.p. $73.2-74.4^{\circ} \mathrm{C}$ (hexane); $[\alpha]_{\mathrm{D}}{ }^{20}-8.0\left(c=0.15, \mathrm{CHCl}_{3}\right)$; IR ( $\mathrm{KBr}$ disk) 3286, 2924, $1045 \mathrm{~cm}^{-1}$; ${ }^{1} \mathrm{H}-\mathrm{NMR} \delta: 5.78(1 \mathrm{H}, \mathrm{m}, \mathrm{H}-7), 4.33(1 \mathrm{H}, \mathrm{d}, \mathrm{J}=11.7 \mathrm{~Hz}, \mathrm{H}-12)$, 3.98-3.85 (4H, m, H-11+H12+2xOH), 3.64 (1H, dd, J= 8.4, 10.7 Hz, H-11), 2.13-1.79 (4H, m), 1.68-1.10 (6H, m), 0.89 (3H, s, 10-Me), 0.86 (3H, s, 4- $\alpha-\mathrm{Me}$ ) , 0.74 (3H, s, 4- $\beta-\mathrm{Me}$ ); ${ }^{13} \mathrm{C}-\mathrm{NMR}$

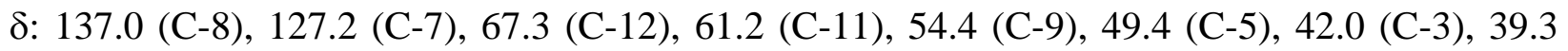
(C-1), 35.6 (C-10), 33.2 (4- $\alpha-\mathrm{Me}), 33.0$ (C-4), 23.6 (C-6), 21.9 (10-Me), 18.8 (C-2), 14.5 (4- $\beta$ Me); microanalysis, calculated for $\mathrm{C}_{15} \mathrm{H}_{26} \mathrm{O}_{2}$ : C, $75.58 \%, \mathrm{H}, 10.99 \%$, found: C, $75.87 \%, \mathrm{H}$, $11.09 \%$.

Preparation of (5S, 10S)-(9 $\alpha H)$-7-drimene-12-tert-butyldiphenylsilyl-11-ol (11). To a solution of diol 9 (1.0 g, $4.19 \mathrm{mmol})$ in dimethylformamide $(30 \mathrm{ml})$, it was slowly added a solution of tert-butyldiphenylsilyl chloride ( $1.15 \mathrm{~g}, 4.2 \mathrm{mmol})$ and imidazole $(0.57 \mathrm{~g}, 8.33 \mathrm{mmol})$ in dimethylformamide $(10 \mathrm{ml})$, with magnetic stirred and under an argon atmosphere. The resulting mixture was stirred for $24 \mathrm{~h}$ at room temperature. It was then diluted with ethyl acetate $(100 \mathrm{ml})$ and washed with water $(50 \mathrm{ml})$. The organic phase was dried $\left(\mathrm{MgSO}_{4}\right)$ and concentrated. After a column chromatography purification, silyl-ether 11 (1.94 g, 97\%) was obtained, as a white solid: m.p. 92.4-93.6 ${ }^{\circ} \mathrm{C}(\mathrm{EtOAc}) ;[\alpha]_{\mathrm{D}}{ }^{18}-25.6\left(c=9.0, \mathrm{CHCl}_{3}\right)$; IR $(\mathrm{KBr}$ disk) 3425, 1956, 1890, 1823, 1741, $1585 \mathrm{~cm}^{-1}$; ${ }^{1} \mathrm{H}-\mathrm{NMR} \delta$ : 7.73-7.68 (4H, m, Ar), 7.44-7.37 (6H, m, Ar), 5.39-5.38 (1H, m, H-7), 4.40 (1H, d, J= 12.0 Hz, H-12), 4.09 (1H, d, J=12.0 Hz, H12), 4.00-3.68 (2H, m, H-11), 2.12-2.11 (1H, m. H-9), 2.00 (1H, bd, J=13.0 Hz, H-1), 1.95-1.08 (6H, m), 1.06 (9H, s, 3 x Me-CSi), 0.89 (6H, s, 4- $\alpha-\mathrm{Me}+4-\beta-\mathrm{Me}), 0.78$ (3H, s, 10-Me); ${ }^{13} \mathrm{C}-$ NMR 8: 135.8 (CH-Ar), 135.7 (CH-Ar), 135.4 (C-Ar), 132.8 (C-8), 129.9 (CH-Ar), 128.0 (C-7), 127.8 (CH-Ar), 127.7 (CH-Ar), 69.3 (C-12), 61.2 (C-11), 55.6 (C-9), 49.4 (C-5), 42.1 (C-3), 39.3 
(C-1), 35.8 (C-10), 33.3 (4- $\alpha-\mathrm{Me}), 33.0$ (C-4), 26.8 (Me-CSi), 23.6 (C-6), 22.0 (4- $\beta-\mathrm{Me}), 19.2$ (C-Si), 18.8 (C-2), 14.6 (10-Me); microanalysis, calculated for $\mathrm{C}_{31} \mathrm{H}_{44} \mathrm{O}_{2} \mathrm{Si}: \mathrm{C}, 78.10 \%, \mathrm{H}$, 9.30\%, found: C, $77.82 \%, \mathrm{H}, 9.67 \%$.

\section{Typical procedure for the oxidation with Jones reagent.}

A magnetically stirred and cold $\left(0^{\circ} \mathrm{C}\right)$ solution of the alcohol substrate $(10 \mathrm{mmol})$ in acetone $(50$ $\mathrm{ml}$ ) was titrated with Jones reagent, until the orange-brown color of the reagent persisted for 30 seconds. The reaction mixture was stirred for and additional $1 \mathrm{~h}$ at room temperature, and then isopropanol $(1 \mathrm{ml})$ was added dropwise to destroy any excess of reagent. Water $(100 \mathrm{ml})$ was added, and the suspension was extracted with ethyl acetate, dried $\left(\mathrm{MgSO}_{4}\right)$, concentrated, and purified by column chromatography.

(4aS,8aS)-2,5,5,8a-Tetramethyl-4a,5,6,7,8,8a-hexahydro-4H-naphthalen-1-one (6). Yield: 65\%; white solid; m.p. 80.0-82.7 ${ }^{\circ} \mathrm{C}$ (EtOAc); $[\alpha]_{\mathrm{D}}{ }^{18}-74.4\left(c=10.1, \mathrm{CHCl}_{3}\right)$; IR $(\mathrm{KBr}$ disk) 2923, $1663 \mathrm{~cm}^{-1}$; ${ }^{1} \mathrm{H}-\mathrm{NMR}$ \&: 6.68-6.65 (1H, m, H-3), 2.31-2.23 (2H, m, H-4), 1.87 (1H, bd, $\mathrm{J}=13.4 \mathrm{~Hz}, \mathrm{H}-8), 1.72$ (3H, d, J=2.0 Hz, 2-Me), 1.69-1.14 (6H, m), 1.01 (3H, s, 8a-Me), 0.96 (3H, s, 5- $\beta-\mathrm{Me}$ ) , 0.88 (3H, s, 5- $\alpha-\mathrm{Me}) ;{ }^{13} \mathrm{C}-\mathrm{NMR} \delta: 205.9$ (C-1), 143.4 (C-3), 132.9 (C-2), 49.4

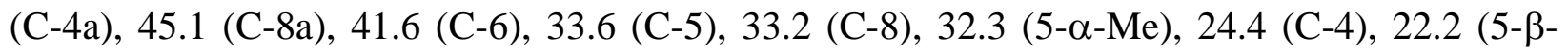
Me), 18.2 (C-7), 17.2 (8a-Me), 16.4 (2-Me); microanalysis, calculated for $\mathrm{C}_{14} \mathrm{H}_{22} \mathrm{O}$ : C, 81.50\%, H, 10.75\%, found: C, 82.04\%, H, 11.12\%.

(4aS,8aS)-2-(tert-Butyl-diphenyl-silanyloxymethyl)-5,5,8a-trimethyl-4a,5,6,7,8,8ahexahydro-4H-naphthalen-1-one (12). Yield: 49\%; colorless oil; $[\alpha]_{\mathrm{D}}{ }^{18}-24.5(c=12.6$, $\mathrm{CHCl}_{3}$ ); IR (film) 1953, 1877, 1823, 1740, $1670 \mathrm{~cm}^{-1}$; ${ }^{1} \mathrm{H}-\mathrm{NMR}$ $8: 7.70-7.66$ (4H, m, Ar), 7.447.35 (6H, m, Ar), 7.09-7.07 (1H, m, H-3), 4.43-4.40 (2H, m, $\left.\mathrm{CH}_{2}-\mathrm{O}\right), 2.45-2.30$ (2H, m, H-4), 1.85 (1H, bd, J=13.4 Hz, H-8), 1.64-1.11 (6H, m), 1.11 (9H, s, 3 x Me-CSi), 1.03 (3H, s, 5- $\alpha-$ Me), ${ }^{*} 1.02$ (3H, s, 5- $\left.\beta-\mathrm{Me}\right),{ }^{*} 1.01$ (3H, s, 8a-Me); ${ }^{13} \mathrm{C}-\mathrm{NMR} \delta: 204.8$ (C-1), 142.1 (C-3), 135.5 (CH, o-Ar), 135.4 (C, Ar), 129.7 (CH, p-Ar), 127.7 (CH, m-Ar), 61.5 ( $\left.\mathrm{CH}_{2}-\mathrm{O}\right), 49.2$ (C-4a), 45.1 (C-8a), 41.6 (C-6), 33.7 (C-5), 32.9 (C-8), 32.4 (5- $\alpha-\mathrm{Me}), 27.0$ (Me-CSi), 24.2 (C-4), 22.3 (5- $\beta$ Me), 19.4 (C-Si), 18.1 (C-7), 17.2 (8a-Me).

Typical procedure for the oxidation with pyridinium chlorochromate. To a magnetically stirred solution of the alcohol substrate $(10 \mathrm{mmol})$ in dichloromethane $(50 \mathrm{ml})$, pyridinium chlorochromate (2.16 g, 10mmol) was added. The brown suspension was stirred overnight, until thin layer chromatography analysis showed the disappearance of the starting material. Celite (500 mg) and ethyl acetate (50 ml) were added to the reaction mixture, and the slurry was filtered through a short pad of silica gel, washing copiously with ethyl acetate. The filtrate was evaporated, and the residue, chromatographed.

(4aS, 8aS)-3,4a,8,8-Tetramethyl-4a,5,6,7,8,8a-hexahydro-1H-naphthalen-2-one (1). Yield: 71\%; colorless oil; $[\alpha]_{\mathrm{D}}{ }^{18}-7.1\left(c=18.2, \mathrm{CHCl}_{3}\right)$; IR (film) 2956, 1704, $1673 \mathrm{~cm}^{-1} ;{ }^{1} \mathrm{H}-\mathrm{NMR} \delta$ : 6.37 (1H, d, J= 1.3 Hz, H-4), 2.52-2.23 (2H, m, H-1), 1.69 (3H, d, J=1.3 Hz, 3-Me), 1.68-1.10 (6H, m), 1.05 (3H, s, 4a-Me), 0.88 (3H, s, 8- $\beta-\mathrm{Me}), 0.85$ (3H, s, 8- $\alpha-\mathrm{Me}) ;{ }^{13} \mathrm{C}-\mathrm{NMR} \delta: 201.6$ 
(C-2), 158.3 (C-4), 131.2 (C-3), 50.6 (C-8a), 41.2 (C-7), 38.4 (C-5), 36.9 (C-4a), 35.4 (C-1), 32.8 (C-8), 32.2 (8- $\alpha-\mathrm{Me}), 20.9$ (8- $\beta-\mathrm{Me}), 18.6$ (C-6), 18.5 (4a-Me), 15.5 (3-Me).

(4aS, 8aS)-5,5,8a-Trimethyl-3-oxo-3,4,4a,5,6,7,8,8a-octahydro-naphthalene-2-carbaldehyde (3). Yield: 55\%; white solid; m.p. 96.9-97.6 ${ }^{\circ} \mathrm{C}$ (hexane); $[\alpha]_{\mathrm{D}}{ }^{18}+36.2\left(c=5.53, \mathrm{CHCl}_{3}\right)$; IR $(\mathrm{KBr}$ disk) 2917, 1681, $1605 \mathrm{~cm}^{-1}$; ${ }^{1} \mathrm{H}-\mathrm{NMR} \delta$ : 10.06 (1H, s, CHO), 7.44 (1H, s, H-4), 2.60 (1H, dd: $\mathrm{J}=4.3,17.6 \mathrm{~Hz}, \mathrm{H}-1), 2.44$ (1H, dd: $\mathrm{J}=4.3,13.6,17.6 \mathrm{~Hz}, \mathrm{H}-1), 1.81-1.06$ (7H, m), 1.16 (3H, s, 4a-Me), 0.94 (3H, s, 8- $\alpha-\mathrm{Me}$ ), 0.92 (3H, s, 8- $\beta-\mathrm{Me}$ ); ${ }^{13} \mathrm{C}-\mathrm{NMR} \delta$ : 205.9 (C-1), 143.4 (C-3), 132.9 (C-2), 49.4 (C-4a), 45.1 (C-8a), 41.6 (C-6), 33.6 (C-5), 33.2 (C-8), 32.3 (5- $\alpha-\mathrm{Me}), 24.4$ (C-4), 22.2 (5- $\beta-\mathrm{Me}), 18.2$ (C-7), 17.2 (8a-Me), 16.4 (2-Me); microanalysis, calculated for $\mathrm{C}_{14} \mathrm{H}_{20} \mathrm{O}_{2}$ : C, 76.33\%, H, 9.15\%, found: C, 75.64\%, H, 9.51\%.

(4aS, 8aS)-5,5,8a-Trimethyl-1-oxo-1,4,4a,5,6,7,8,8a-octahydro-naphthalene-2-carbaldehyde (7). Yield: 66\%; yellow solid; $[\alpha]_{\mathrm{D}}{ }^{18}+26.6\left(c=0.94, \mathrm{CHCl}_{3}\right)$; IR (KBr disk) 2932, 1712, 1647 $\mathrm{cm}^{-1}$; ${ }^{1} \mathrm{H}-\mathrm{NMR} \delta$ : 10.00 (1H, s, CHO), 7.80-7.75 (1H, m, H-3), 2.67 (1H, dt, J= 5.1, $21.1 \mathrm{~Hz}, \mathrm{H}-$ 4), 2.45 (1H, ddd, J= 2.5, 11.0, $21.1 \mathrm{~Hz}, \mathrm{H}-4), 2.00$ (1H, dd, J=2.5, $11.0 \mathrm{~Hz}, \mathrm{H}-4 \mathrm{a}), 2.07-1.20$ $(6 \mathrm{H}, \mathrm{m}), 1.09$ (3H, s, 8a-Me), 1.01 (3H, s, 5- $\beta-\mathrm{Me}), 0.94$ (3H, s, 5- $\alpha-\mathrm{Me}) ;{ }^{13} \mathrm{C}-\mathrm{NMR} \delta: 203.4$ (C-1), 189.9 (CHO), 156.2 (C-3), 132.8 (C-2), 48.4 (C-4a), 45.3 (C-8a), 41.4 (C-6), 33.7 (C-5), 32.6 (C-8), 32.2 (5- $\alpha-\mathrm{Me}), 25.2$ (C-4), 22.1 (5- $\beta-\mathrm{Me}), 17.9$ (C-7), 16.8 (8a-Me); microanalysis, calculated for $\mathrm{C}_{14} \mathrm{H}_{20} \mathrm{O}_{2}$ : C, 76.33\%, H, 9.15\%, found: C, 76.52\%, H, 9.60\%.

(4aS,8aS)-(4aS,8aS)-3-(tert-Butyl-diphenyl-silanyloxymethyl)-4a,8,8-trimethyl-4a,5,6,7,8,8ahexahydro-1H-naphthalen-2-one (14) .Yield: 83\%; yellow oil; $[\alpha]_{\mathrm{D}}{ }^{20}-4.24$ (c= 11.8, $\mathrm{CHCl}_{3}$ ); IR (film) 2930, 1704, 1668, 1113, $702 \mathrm{~cm}^{-1}$; ${ }^{1} \mathrm{H}-\mathrm{NMR} \underline{\underline{\delta}}$ : 7.65-7.61 (4H, m, Ar); 7.41-7.32 (6H, m, Ar); $6.76(1 \mathrm{H}, \mathrm{t}, \mathrm{J}=2.0 \mathrm{~Hz}, \mathrm{H}-4) ; 4.40$ (2H, d, J= $\left.1.7 \mathrm{~Hz}, \mathrm{CH}_{2}-\mathrm{O}\right) ; 2.38-2.30$ (2H, m, H-1); 1.68-1.19 (7H, m); 1.07 (9H, s, $\left.3 \mathrm{CH}_{3}-\mathrm{CSi}\right) ; 1.06$ (3H, s, 4a-Me), 0.90 (3H, s, 8- $\left.\beta-\mathrm{Me}\right), 0.86$ (3H, s, 8- $\alpha-\mathrm{Me}$ ); ${ }^{13} \mathrm{C}-\mathrm{NMR} \delta$ : 200.6 (C-7); 156.8 (C-9); 135.6 (CH-Ar); 135.5 (CH-Ar); 134.0 (C-8); 133.5 (Cq-Ar); 133.5 (Cq-Ar); 129.7 (CH-Ar); 127.7 (CH-Ar); 60.6 (C-12); 50.4 (C-5); 41.3 (C-3); 38.4 (C-1); 36.5 (C-10); 35.6 (C-6); 32.9 (C-4); 32.3 (4- $\alpha \mathrm{Me}$ ); 26.9 (Me-CSi); 20.9 (4- $\beta \mathrm{Me}) ; 19.3$ (Cq-Si).

Typical procedure for the deprotection of tert-butyldiphenylsilyl ethers. To a magnetically stirred solution of the silyl ether substrate $(1 \mathrm{mmol})$ in dry tetrahydrofuran $(20 \mathrm{ml})$, a $1 \mathrm{M}$ tetrahydrofuran solution of tetrabutylammonium fluoride $(2.3 \mathrm{ml}, 0.23 \mathrm{mmol})$ was added. The reaction mixture was stirred for $2 \mathrm{~h}$ at room temperature, diluted with ethyl acetate $(50 \mathrm{ml})$, washed with $5 \%$ hydrochloric acid $(20 \mathrm{ml})$, saturated sodium bicarbonate solution $(50 \mathrm{ml})$, dried $\left(\mathrm{MgSO}_{4}\right)$ and evaporated. The residue was finally chromatographed.

(4aS,8aS)-2-Hydroxymethyl-5,5,8a-trimethyl-4a,5,6,7,8,8a-hexahydro-4H-naphthalen-1-one (13). Yield: 95\%; colorless oil; $[\alpha]_{\mathrm{D}}{ }^{18}-61.7\left(c=6.23, \mathrm{CHCl}_{3}\right)$; IR (film) 3444, $1667 \mathrm{~cm}^{-1} ;{ }^{1} \mathrm{H}-$ NMR $\delta$ : 6.88-6.84 (1H, m, H-3), 4.18 (2H, s, $\left.\mathrm{CH}_{2}-\mathrm{O}\right), 2.81$ (1H, bs, OH), 2.38-2.27 (2H, m, H4), 1.89-1.80 (1H, m, H-8), 1.65-1.13 (6H, m), 1.05 (3H, s, 8a-Me), 0.96 (3H, s, 5- $\alpha-\mathrm{Me}), 0.89$ (3H, s, 5- $\beta-\mathrm{Me}) ;{ }^{13} \mathrm{C}-\mathrm{NMR} \delta$ : 206.7 (C-1), 145.1 (C-3), 135.8 (C-2), $62.4\left(\mathrm{CH}_{2}-\mathrm{O}\right), 49.0$ (C-4a), 


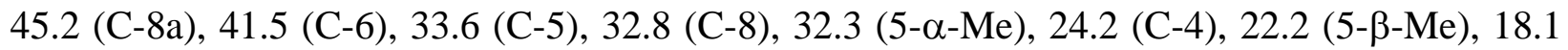
(C-7), 17.1 (8a-Me).

(4aS,8aS)-3-Hydroxymethyl-4a,8,8-trimethyl-4a,5,6,7,8,8a-hexahydro-1H-naphthalen-2-one (15). Yield: 92\%; colorless oil; $[\alpha]_{\mathrm{D}}{ }^{18}+2.56\left(c=11.7, \mathrm{CHCl}_{3}\right)$; IR (film) 3423, $1668 \mathrm{~cm}^{-1} ;{ }^{1} \mathrm{H}-$ NMR $\delta: 6.54$ (1H, s, H-4), 4.16 (2H, s, $\left.\mathrm{CH}_{2}-\mathrm{O}\right), 2.84$ (1H, bs, OH), 2.48-2.24 (2H, m, H-1), 1.74-1.16 (7H, m), 1.06 (3H, s, 4a-Me), 0.88 (3H, s, 8- $\alpha-\mathrm{Me}), 0.85$ (3H, s, 8- $\beta-\mathrm{Me}) ;{ }^{13} \mathrm{C}-\mathrm{NMR} \delta$ : 202.3 (C-2), 158.9 (C-4), 133.9 (C-3), 61.4 ( $\left.\mathrm{CH}_{2}-\mathrm{O}\right), 50.3$ (C-8a), 41.1 (C-7), 38.0 (C-5), 36.7 (C-4a), 35.6 (C-1), 32.9 (C-8), 32.2 (8- $\alpha-\mathrm{Me}), 20.9$ (8- $\beta-\mathrm{Me}), 18.5$ (C-5), 18.3 (4a-Me).

\section{Acknowledgments}

The authors thank Facultad de Química de la Pontificia Universidad Católica de Chile, and DIPUC (Dirección de Investigaciones de la Pontificia Universidad Católica de Chile, Research Grant No 98-10E) for financial support. M. A. C. and L. E. M. thank CONICYT (Consejo Nacional de Investigaciones Científicas y Tecnológicas) and DIPUC, for their fellowships.

\section{References}

1. Jansen, B. M. J.; de Groot, A. Nat. Prod. Rep. 1991, 8, 309.

2. Van Beek, T. A.; de Groot, A. Rcl. Trav. Chim. Pays Bas. 1986, 105, 513, and references cited therein.

3. Anke, H.; Sterner, O. Planta Med. 1991, 57, 344, and references cited therein.

4. Asakawa, Y.; Dawson, G. W.; Griffiths, D. C.; Lallemand, J. Y.; Ley, S. V.; Mori, K.; Mudd, A.; Pezechk-Leclaire, M.; Pickett, J. A.; Watanabe, H.; Woodcock, C. M.; Zhong-Ning, Z. J. Chem Ecol. 1988, 14, 1845.

5. Marston, A.; Hostettmann, K. Phytochemistry 1985, 24, 639.

6. Fukuyama, Y.; Sato, T.; Asakawa, Y.; Takemoto, T. Phytochemistry 1982, 21, 2895.

7. Jansen, B. M. J.; de Groot, A. Nat. Prod. Rep. 1991, 8, 319

8. Hlubucek, J. R.; Aasen, A. J.; Kimland, B.; Enzell, C. R. Phytochemistry 1973, 12, 2555.

9. Fukuyama, Y.; Sato, T.; Miura, I.; Asakawa, Y. Phytochemistry 1985, 24, 1521.

10. Asakawa, Y.; Takemoto, T. Experientia 1979, 35, 1420.

11. Iwamoto, M.; Ohtsu, H.; Matsunaga, S.; Tanaka, R. J. Nat. Prod. 2000, 63, 1381.

12. Hersel, U.; Steck, M.; Seifert, K. Eur. J. Org. Chem. 2000, 1609.

13. de Groot, A.; Jansen, B. J. M.; Verstegen-Haaksma, A. A.; Orru, R. V. A.; Stork, G. A.; Wijnberg, J. B. P. A. Pure \& Appl. Chem. 1994, 66, 2053.

14. Jonassohn, M.; Davidsson, R.; Kahnberg, P.; Sterner, O. Tetrahedron 1997, 53, 237. 
15. Appel, H. H.; Brooks, C. J. W.; Overton, K. H. J. Chem Soc(C) 1959, 3323, and references cited therein.

16. Peña, W.; López, J. T.; Cortés, M. Synth. Commun. 1989, 19, 2841.

17. Cortés, M.; Razmilic, I.; López, J. J. Nat. Prod. (Lloydia) 1990, 53, 1369.

18. Oyarzún, M. L.; Cortés, M.; Sierra, J. Synth. Commun. 1982, 12, 951.

19. El Sayah, M.; Cechinel Filho, V.; Yunes, R. A.; Pinheiro, T. R.; Calixto, J. B. Eur. J. Pharm. 1998, 344, 215.

20. Cechinel Filho, V.; Schlemper, V.; Santos, A. R. S.; Pinheiro, T. R.; Yunes, R. A.; Mendes, G. L.; Calixto, J. B.; Delle Monache, F. J. Ethnopharm. 1998, 62, 223.

21. Brooks, C. J. W.; Lindsay, G.; Overton, K. H. J. Chem. Soc. (C) 1970, 203.

22. Hlubucek, J. R.; Aasen, A. J.; Almquist, S.; Enzell, C. R. Acta Chem. Scand. 1974, B28, 18.

23. Cheung, W. S.; Wong, H. N. C. Tetrahedron Lett. 1998, 39, 6521.

24. Hollinshead, D. M.; Howell, S. C.; Ley, S. V.; Mahon, M.; Ratcliffe, N. J. Chem. Soc., Perkin Trans. 1 1983, 1579.

25. Sierra, J. R.; López, J. T., Cortés, M. J. Phytochemistry 1986, 25, 253. 